Check for updates

Cite this: Chem. Commun., 2017, 53,6516

Received 5th April 2017

Accepted 25th May 2017

DOI: $10.1039 / \mathrm{c} 7 \mathrm{cc} 02631 \mathrm{e}$

rsc.li/chemcomm

\section{Uniform distribution of post-synthetic linker exchange in metal-organic frameworks revealed by Rutherford backscattering spectrometry $\dagger$}

\author{
Ulrike Fluch, ${ }^{a}$ Valentina Paneta, (D) ${ }^{b}$ Daniel Primetzhofer (D) $*^{b}$ and Sascha Ott (D) $*^{a}$
}

\begin{abstract}
Rutherford backscattering spectrometry (RBS) has been used for the first time to study post-synthetic linker exchange (PSE) in metalorganic frameworks. RBS is a non-invasive method to quantify the amount of introduced linker, as well as providing a means for depth profiling in order to identify the preferred localization of the introduced linker. The exchange of benzenedicarboxylate (bdc) by similarly sized 2-iodobenzenedicarboxylate (I-bdc) proceeds considerably slower than migration of I-dbc through the UiO-66 crystal. Consequently, the I-bdc is found evenly distributed throughout the UiO-66 samples, even at very short PSE exposure times.
\end{abstract}

Metal-organic frameworks (MOFs) are three-dimensional coordination polymers that consist of metal or metal cluster secondary building units (SBUs) that are interconnected by organic linker molecules. Traditional applications of these highly porous and large surface area materials have been in gas storage and separation, ${ }^{1-6}$ but also more high-end applications such as light harvesting ${ }^{7-10}$ or catalysis ${ }^{11-14}$ have emerged more recently.

Although MOFs are stable crystalline materials, it has been shown in many cases that the constituting metals in the SBUs, as well as the organic linker molecules can be exchanged by postsynthetic methods. ${ }^{15-22}$ Post-synthetic linker exchange (PSE), also termed solvent-assisted linker exchange (SALE), is particularly useful for the integration of linkers that are not compatible with the often rather harsh solvothermal growth conditions. Illustrating the power of PSE, Choe et al. ${ }^{23}$ reported the complete replacement of all linkers, while Liu et al. described the introduction of different linkers into one framework by PSE. ${ }^{24}$ It is noteworthy that PSE occurs within intact MOFs rather than by dissolving and recrystallization. ${ }^{25}$

Despite its growing popularity, little is known about the exact mechanism of PSE. At first glance, it is intuitive to assume

\footnotetext{
${ }^{a}$ Department of Chemistry - Ångström Laboratory, Uppsala University, Box 523, 75120 Uppsala, Sweden.E-mail: sascha.ott@kemi.uu.se

${ }^{b}$ Department of Physics and Astronomy, Uppsala University, Box 516,

75120 Uppsala, Sweden. E-mail: daniel.primetzhofer@physics.uu.se

$\dagger$ Electronic supplementary information (ESI) available. See DOI: 10.1039/c7cc02631e
}

that PSE initially occurs at the surface of the crystals where the MOF is in contact with the solution of the new linker. On the other hand, such a preference would require a PSE rate that is higher than the migration of free linkers through the crystals. Based on these considerations, it is unclear whether PSE occurs initially at the periphery, or evenly distributed throughout the MOF crystals. Investigations of this aspect of PSE in MOFs have hitherto been hampered by the shortage of suitable analytical methods.

$\mathrm{X}$-ray photoelectron spectroscopy (XPS) is a commonly used technique to study the near-surface composition of a material. Its information depth of typically $10 \mathrm{~nm}$ is, however, not suitable for depth-profiling of intact MOF crystals with up to $\mu \mathrm{m}$ dimensions. Energy-dispersive X-ray spectroscopy (EDS, EDX, or XEDS) is also commonly used, in particular for the analysis of cross sections, e.g. in transmission electron microscopy (TEM). Both methods, XPS and EDX, however, suffer from similar drawbacks such as limited and not well-defined sampling volumes that result in very limited depth resolution and uncertain information depth, mainly due to the limited inelastic mean free path of electrons in the relevant energy regimes. This fact also introduces strong dependencies on the material density and the electron energies detected or used in the excitation process. Sputtering of samples in electron-based analysis processes is an option, however, a number of difficulties that arise from sample geometry, different sputter rates for the constituents and intermixing of the material can be expected, and advanced sputtering equipment and data analysis would be required to establish depth profiles. ${ }^{26}$ Alternatively, time-of-flight secondary ion mass spectrometry (TOF-SIMS) has recently been applied to determine the exchange rate of linkers in thin MOF films, but no depthprofiling was reported. ${ }^{25}$

Rutherford backscattering spectrometry (RBS) is one of the most commonly used nuclear techniques in the ion beam analysis (IBA) field. ${ }^{27}$ It is typically employed for the study of the nearsurface composition of materials such as thin film stacks, using an accelerated ion beam. ${ }^{28}$ Identification, quantification and depth distribution of an element in a sample is achieved by detecting the energy of the beam particles after they have been elastically 
scattered by the Coulomb potential of the sample nuclei (Rutherford scattering). The elastic scattering provides information on the mass of the involved scattering partners which is element specific. In addition, the beam particles lose energy while crossing the sample material before and after the point of being scattered, providing a depth perception. Using an alpha particle beam, RBS typically concerns the analysis of heavy nuclei with $A>30$ when applied in such scattering cases. The choice of different primary particles permits the change of the mass and depth resolution depending on sample requirement. With ion energies of a few $\mathrm{MeV}$, the typical information depth is in the order of a few $\mu \mathrm{m}$ below the surface, i.e. the scale of most MOF particles. ${ }^{29}$ In this paper, we will for the first time use RBS for the quantitative analysis of PSE and as a means to detect whether PSE proceeds statistically throughout the UiO-66 crystals.

UiO-66 $(\mathrm{Zr})^{30}$ is composed of $\mathrm{Zr}_{6} \mathrm{O}_{4}(\mathrm{OH})_{4}$ nodes that are bridged by twelve benzene-1,4-dicarboxylates (bdc) linkers, and was chosen for this study on grounds of its chemical stability ${ }^{31}$ and welldocumented propensity to engage in PSE. 2-Iodobenzene-1,4dicarboxylate (I-bdc) was chosen as exchanging linker as iodine is a suitable heavy element for RBS analysis. PSE was performed in both bulk UiO-66 suspensions, as well as in UiO-66 that was grown on silicon wafers (UiO-66@Si). The former has the advantage that PSE can be performed on larger scales, and RBS quantification of introduced linkers can be compared to yields obtained for digested samples by ${ }^{1} \mathrm{H}$ NMR spectroscopy. For the RBS measurements, bulk UiO-66 samples were drop-cast onto silicon wafers which gave rise to multiple-layers aggregates as evidenced by SEM (Fig. S8, ESI $\dagger$ ).

In UiO-66@Si, UiO-66 grows as a single crystal monolayer with a high preference for the (111) phase, ${ }^{32}$ as evidenced by the PXRD pattern of the UiO-66@Si samples in which some peaks of the bulk UiO-66 pattern are missing (Fig. S7, ESI†). By having UiO-66 immobilized on the silicon substrates, exposure of UiO-66@Si to the PSE conditions can be kept on the timescales of seconds. Also, one of the sides of the MOF crystals is blocked by the substrate which could affect the formation of a gradient during PSE. Finally the well-defined geometry of the UiO-66@Si samples is advantageous for RBS analyses.

Irrespective of whether PSE was performed in bulk or silicongrown UiO-66, RBS experimental conditions were tuned in order to not compromise the crystallinity of the samples, and sample morphology and crystallinity is unchanged during PSE, as evidenced by SEM (Fig. S9, ESI†).

In the first set of experiments, the suitability of RBS as a method to quantify PSE was evaluated. Fig. 1a shows typical RBS spectra of Si-deposited, bulk UiO-66 samples that had been exposed to PSE conditions for five minutes to up to three hours. Visible are the number of beam particles scattered by the iodine nuclei in the introduced I-bdc linker, normalized to those scattered by zirconium nuclei of the MOFs (inset Fig. 1a). The energy distribution of the detected particles is the result of the energy losses that arise from beam penetration into the UiO-66 sample and can be converted to depth scale (see ESI $\dagger$ ). The signal width is relatively broad as one would expect from an agglomerated drop-cast sample. From an integration of the iodine peak area relative to the normalized $\mathrm{Zr}$ signal (inset Fig. 1a), and taking
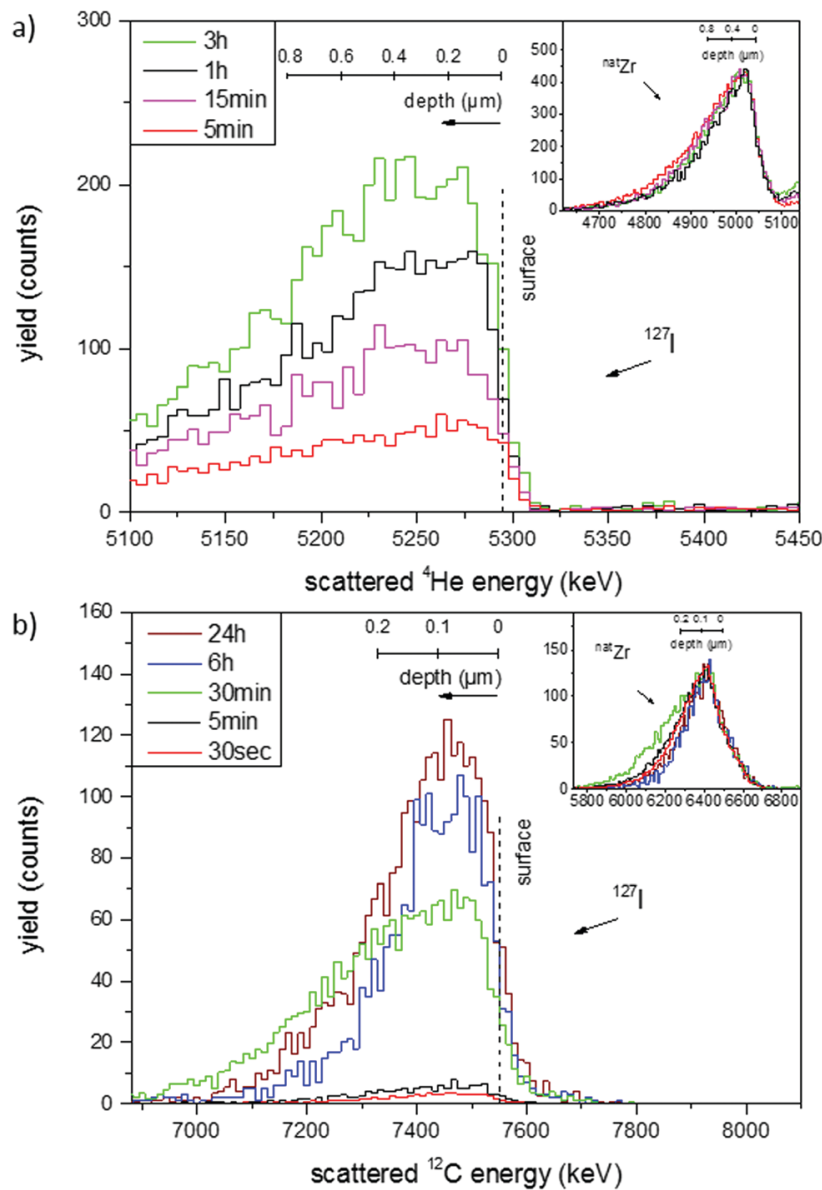

c)

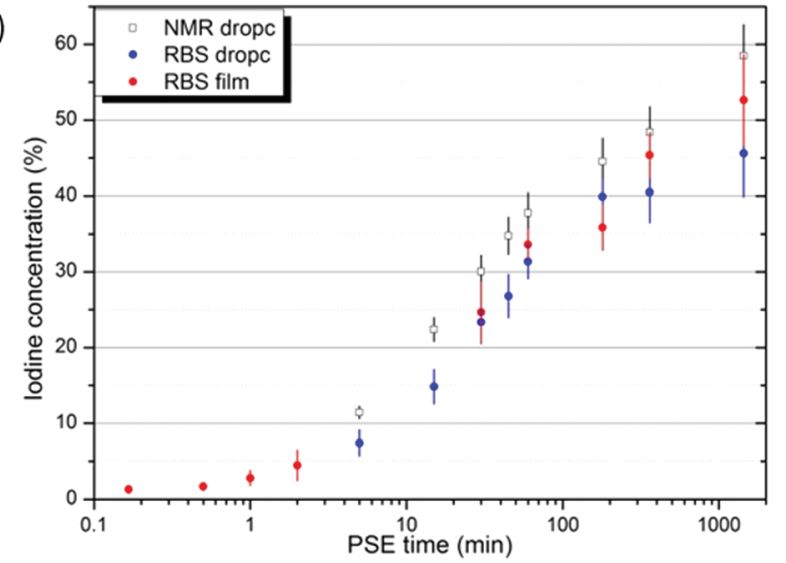

Fig. 1 (a) RBS spectra (6 MeV particle energy, beam of ${ }^{4} \mathrm{He}$ ) of Si-deposited samples of bulk UiO-66 which were exposed to PSE conditions for time periods as indicated. Shown is the response from the beam particles scattered by iodine; inset: normalized zirconium reference signals. (b) RBS spectra (11 MeV particle energy, beam of ${ }^{12} \mathrm{C}$ ) of UiO-66@Si samples which were exposed to PSE conditions for time periods as indicated. (c) Logarithmic plot of the concentration of introduced I-bdc vs. PSE time. Black squares are the results from ${ }^{1} \mathrm{H}$ NMR measurements, (relative to the total linker amount). The blue and red dots are the results from the RBS experiments of the drop-cast bulk and UiO-66@Si samples, respectively (given relative to the $\mathrm{Zr}$ content).

into account the different scattering probabilities of the two nuclei, it is possible to quantify the I-bdc content in the samples and thus the degree of PSE. 
The PSE quantifications obtained from RBS was validated in comparison to ${ }^{1} \mathrm{H}$ NMR yields obtained from digested samples. As shown in Fig. 1c, the I-bdc concentrations as determined by RBS correlate well with those obtained by ${ }^{1} \mathrm{H}$ NMR, but are generally somewhat lower. In order to explain this discrepancy, it is important to realize that RBS analysis gives I-bdc concentrations relative to the amount of $\mathrm{Zr}$, while those determined by NMR are relative to the total linker content. The two techniques will give different results when alternative ligands like hydroxide ions coordinate to some $\mathrm{Zr}$ centers instead of bdc. The information obtained by the two techniques is thus somewhat complementary: as the $\mathrm{Zr}$ centers are integral to the UiO-66, RBS quantification gives a more realistic picture of the actual I-bdc content in the framework, while NMR measurements can reveal the type of linkers that have been exchanged. More specifically, the NMR studies revealed that initial incorporation of I-bdc proceeds preferentially at the defect sites, i.e. at SBUs that are ligated by benzoate that was the modulator in the UiO-66 synthesis (Fig. S1, ESI $\dagger$ ). After 30 minutes, the amount of I-bdc increases on the expense of bdc, proving that the high percentage introduction of I-bdc is a true PSE process.

Ligand exchange was also probed in UiO-66@Si samples that were exposed to PSE conditions for shorter periods of time down to five seconds (Fig. 1b). $\ddagger$ The single crystal monolayer of the UiO-66@Si samples give rise to RBS spectra at the iodine edge that are considerably narrower in shape compared to those of the drop-cast, agglomerated bulk samples (Fig. S6, ESI $\dagger$ ). While the RBS-determined incorporation yields in UiO-66@Si samples cannot be correlated with NMR yields due to sample scarcity, it is evident from Fig. 1c that they correlate well with the PSE yields from bulk UiO-66 PSE experiments. On the PSE timescale of seconds, I-bdc incorporation is as low as $2 \%$, while after 24 hours, about 55\% exchange has occurred.

As the energy distribution of the RBS spectra contains a depth profile, the iodine signals of all spectra were normalized and their shape compared. This analysis can in principle be done for the drop-cast bulk samples, but the heterogeneity of the films convolutes these analyses. The crystal monolayer samples of UiO-66@Si give unambiguous results, and were therefore used for this study. As representatively shown for two UiO-66@Si samples (after $30 \mathrm{~s}$ and $24 \mathrm{~h}$ PSE exposure time) in Fig. 2 (solid lines), it is clear that the normalized RBS signals of iodine are basically identical. Moreover, the iodine signals for the two samples are identical to that of a reference sample where UiO-66 was grown solvothermally with the I-bdc linker on silicon (UiO-66-(I-bdc)@Si). Finally, all three signals are also very similar in shape and width to that of the corresponding zirconium signals. Together, all three lines of evidence strongly support the conclusion that the introduced I-bdc linkers are uniformly distributed throughout the crystals.

The absence of any detectable depth distribution of the introduced I-bdc linker points towards a PSE rate that is slower than I-bdc transport through the crystals. This is the case for I-bdc incorporation by "true" PSE, as well as for its integration at defect sites which seems to be the predominant process at the beginning of the PSE experiments (vide supra). In other words,

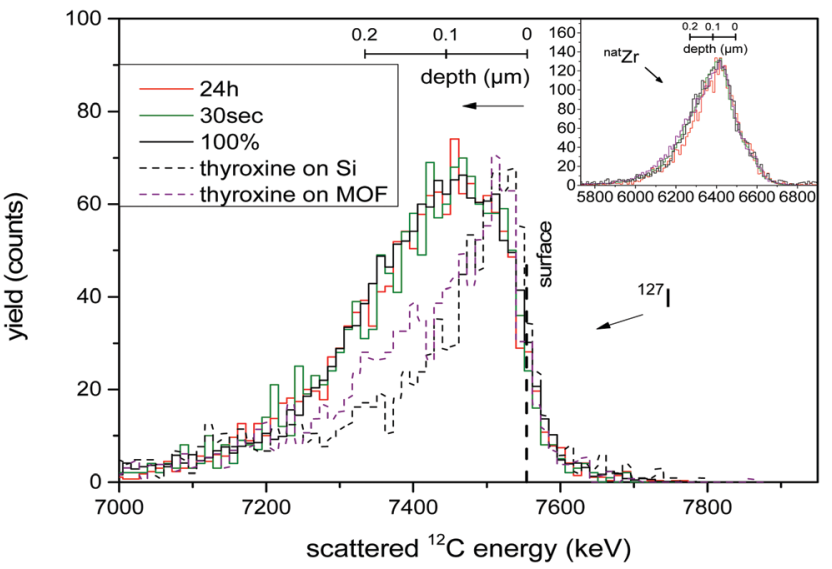

Fig. 2 Normalized RBS signal of the iodine content and normalized RBS zirconium signal (inset). The vertical dashed line marks the surface of the sample, while the lower energies correspond to the depth of the sample with the corresponding depth scales depicted. The solid lines correspond to UiO-66@Si samples that have been exposed to PSE for times as indicated, $100 \%$ is the (UiO-66-(l-bdc) aSi) sample, and the dotted lines correspond to reference samples with thyroxine.

even the occupancy of defect sites in UiO-66@Si is slower than Ibdc transport through the crystal.

The fast transport of I-bdc through the UiO-66 pores is facilitated by its small size, and should be impeded for larger compounds. To probe this hypothesis, and to test RBS as a method that can detect depth gradients, a concentrated methanolic solution of thyroxine was spin-cast onto UiO-66@Si as well as onto pristine silicon wafers. Thyroxine $\left(\mathrm{C}_{15} \mathrm{H}_{11} \mathrm{I}_{4} \mathrm{NO}_{4}\right)$ is a nanometersized thyroid hormone that is larger than the UiO-66 pore windows of $c a .6 \AA^{33}$ It contains four iodine substituents as RBS probes in addition to a carboxylate group for UiO-66 binding.

As expected, the $\mathrm{Zr}$ peak in the thyroxine@UiO-66@Si sample is not affected by the casting process and the thyroxine is identified by a peak in the iodine energy region in both thyroxine@Si and thyroxine@UiO-66@Si (Fig. 2, dotted lines). Normalizing the signal height of the two spectra to those of the PSE samples, a clear difference between the RBS responses becomes evident. The two sets of iodine signals differ both in spectral width as well as peak position. The peak positions closer to the theoretical high-energy ${ }^{127}$ I edge in the thyroxine samples is a clear indication for iodine centres that are confined to a thinner space close to the sample surface as compared to the situation in the PSE samples. This finding is consistent with a confinement of thyroxine at the very surface of thyroxine@Si and thyroxine@UiO-66@Si, a conclusion that is further supported by the narrower peak shape compared to that in the PSE samples. As expected, the width of the signal corresponding to iodine in the pure thyroxine@Si is also narrower compared to that in thyroxine@UiO66@Si due to the rougher surface of the latter and the fact that thyroxine is expected to cover also the side facets of the crystals. These thyroxine molecules will only be available for scattering for ions which have already penetrated a part of the crystal, thus mimicking a depth distribution despite of being located at the crystal surface. All-in-all, the thyroxine experiments prove that RBS has the potential to reveal differences in depth distribution. 
In the present paper, we have shown for the first time that RBS can be used as a non-destructive quantification method to determine the yield of PSE reactions in a metal-organic framework. As RBS analysis uses the Zr centers as an internal standard, highly reliable concentrations of the newly introduced linkers in the framework can be obtained. This information cannot be deduced directly from ${ }^{1} \mathrm{H}$ NMR analysis of digested samples. In addition, RBS analysis can be performed on small sample quantities like UiO-66@Si where other quantification methods are not feasible. Furthermore, it is shown that RBS is a viable method to identify distribution differences of the introduced linkers in the MOF. In the investigated case of bdc being exchanged by I-bdc, RBS allows to draw the conclusion that the migration of I-dbc through the UiO-66 crystal proceeds faster than its incorporation. Consequently, the I-bdc is found statistically distributed throughout the UiO-66 samples, even at very short PSE exposure times down to five seconds. The method provokes further studies of linker diffusion and the kinetics of PSE, in particularly on MOFs that are immobilized on substrates and that are exposed to PSE conditions for short periods of time.

Financial support from the Swiss National Science Foundation for an Early Postdoc. Mobility stipend (U. F.), the Swedish Research Council, the Swedish Energy Agency and the European Research Council (ERC-CoG2015-681895_MOFcat) is gratefully acknowledged. The operation of the tandem accelerator was supported by a research infrastructure fellowship of the Swedish Foundation for Strategic Research (SSF) under contract RIF140053. Mr Andreas Wagner is acknowledged for his contributions in early stages of the project.

\section{Notes and references}

\$ RBS spectra of UiO-66@Si samples were mainly recorded with ${ }^{12} \mathrm{C}^{3+}$ beam at higher beam energy (11 MeV) and are qualitatively comparable to those obtained with ${ }^{4} \mathrm{He}^{+}$beam at $6 \mathrm{MeV}$.

1 J.-R. Li, R. J. Kuppler and H.-C. Zhou, Chem. Soc. Rev., 2009, 38, 1477-1504.

2 T. A. Makal, J.-R. Li, W. Lu and H.-C. Zhou, Chem. Soc. Rev., 2012, 41, 7761-7779.

3 S. Chavan, J. G. Vitillo, D. Gianolio, O. Zavorotynska, B. Civalleri, S. Jakobsen, M. H. Nilsen, L. Valenzano, C. Lamberti, K. P. Lillerud and S. Bordiga, Phys. Chem. Chem. Phys., 2012, 14, 1614-1626.

4 K. Sumida, D. L. Rogow, J. A. Mason, T. M. McDonald, E. D. Bloch, Z. R. Herm, T.-H. Bae and J. R. Long, Chem. Rev., 2012, 112, 724-781.

5 L. J. Murray, M. Dincă and J. R. Long, Chem. Soc. Rev., 2009, 38, 1294-1314.
6 M. P. Suh, H. J. Park, T. K. Prasad and D.-W. Lim, Chem. Rev., 2012, 112, 782-835.

7 C. Y. Lee, O. K. Farha, B. J. Hong, A. A. Sarjeant, S. T. Nguyen and J. T. Hupp, J. Am. Chem. Soc., 2011, 133, 15858-15861.

8 T. Zhang and W. Lin, Chem. Soc. Rev., 2014, 43, 5982-5993.

9 H.-J. Son, S. Jin, S. Patwardhan, S. J. Wezenberg, N. C. Jeong, M. So, C. E. Wilmer, A. A. Sarjeant, G. C. Schatz, R. Q. Snurr, O. K. Farha, G. P. Wiederrecht and J. T. Hupp, J. Am. Chem. Soc., 2013, 135, 862-869.

10 S. Jin, H.-J. Son, O. K. Farha, G. P. Wiederrecht and J. T. Hupp, J. Am. Chem. Soc., 2013, 135, 955-958.

11 J. Lee, O. K. Farha, J. Roberts, K. A. Scheidt, S. T. Nguyen and J. T. Hupp, Chem. Soc. Rev., 2009, 38, 1450-1459.

12 S. Pullen, H. Fei, A. Orthaber, S. M. Cohen and S. Ott, J. Am. Chem. Soc., 2013, 135, 16997-17003.

13 J. S. Seo, D. Whang, H. Lee, S. I. Jun, J. Oh, Y. J. Jeon and K. Kim, Nature, 2000, 404, 982-986.

14 L. Ma, C. Abney and W. Lin, Chem. Soc. Rev., 2009, 38, 1248-1256.

15 H. J. Park, Y. E. Cheon and M. P. Suh, Chem. - Eur. J., 2010, 16, 11662-11669.

16 M. Kondo, S. Furukawa, K. Hirai and S. Kitagawa, Angew. Chem., Int. Ed., 2010, 49, 5327-5330.

17 J. Seo, C. Bonneau, R. Matsuda, M. Takata and S. Kitagawa, J. Am. Chem. Soc., 2011, 133, 9005-9013.

18 M. Kim, J. F. Cahill, H. Fei, K. A. Prather and S. M. Cohen, J. Am. Chem. Soc., 2012, 134, 18082-18088.

19 Z. Wang and S. M. Cohen, Chem. Soc. Rev., 2009, 38, 1315-1329.

20 A. D. Burrows, C. G. Frost, M. F. Mahon and C. Richardson, Angew. Chem., Int. Ed., 2008, 47, 8482-8486.

21 P. Deria, J. E. Mondloch, O. Karagiaridi, W. Bury, J. T. Hupp and O. K. Farha, Chem. Soc. Rev., 2014, 43, 5896-5912.

22 S. M. Cohen, Chem. Rev., 2012, 112, 970-1000.

23 B. J. Burnett, P. M. Barron, C. Hu and W. Choe, J. Am. Chem. Soc., 2011, 133, 9984-9987.

24 C. Liu, T.-Y. Luo, E. S. Feura, C. Zhang and N. L. Rosi, J. Am. Chem. Soc., 2015, 137, 10508-10511.

25 M. C. So, M. H. Beyzavi, R. Sawhney, O. Shekhah, M. Eddaoudi, S. S. Al-Juaid, J. T. Hupp and O. K. Farha, Chem. Commun., 2014, 51, $85-88$.

26 A. J. Taylor, D. J. Graham and D. G. Castner, Analyst, 2015, 140, 6005-6014.

27 Handbook of modern ion beam materials analysis, ed. Y. Wang and M. A. Nastasi, Materials Research Society, Warrendale, PA, 2nd edn, 2009.

28 (a) C. Jeynes, N. P. Barradas and E. Szilagyi, Anal. Chem., 2012, 84, 6061-6069; (b) C. Jeynes and J. L. Colaux, Analyst, 2016, 141, $5944-5985$.

29 A. Wagner, S. Pullen, S. Ott and D. Primetzhofer, Nucl. Instrum. Methods Phys. Res., Sect. B, 2016, 371, 327-331.

30 J. H. Cavka, S. Jakobsen, U. Olsbye, N. Guillou, C. Lamberti, S. Bordiga and K. P. Lillerud, J. Am. Chem. Soc., 2008, 130, 13850-13851.

31 L. Valenzano, B. Civalleri, S. Chavan, S. Bordiga, M. H. Nilsen, S. Jakobsen, K. P. Lillerud and C. Lamberti, Chem. Mater., 2011, 23, 1700-1718.

32 F. M. Hinterholzinger, S. Wuttke, P. Roy, T. Preuße, A. Schaate, P. Behrens, A. Godt and T. Bein, Dalton Trans., 2012, 41, 3899-3901.

33 Q. Yang, A. D. Wiersum, P. L. Llewellyn, V. Guillerm, C. Serre and G. Maurin, Chem. Commun., 2011, 47, 9603-9605. 